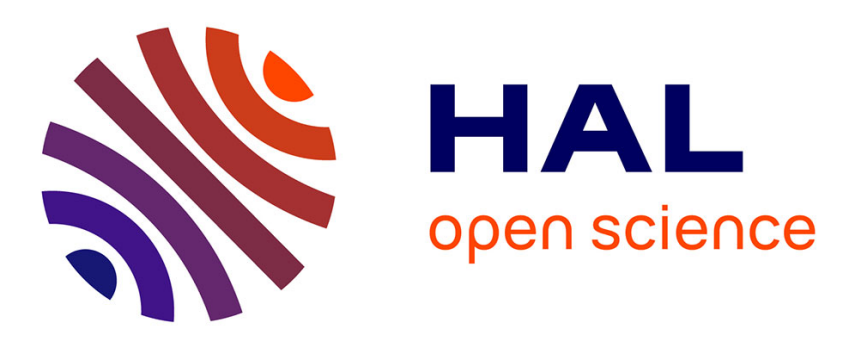

\title{
Sonar image segmentation based on statistical modeling of wavelet subbands
}

\author{
Ayoub Karine, Nour-Eddine Lasmar, Alexandre Baussard, Mohammed El \\ Hassouni
}

\section{- To cite this version:}

Ayoub Karine, Nour-Eddine Lasmar, Alexandre Baussard, Mohammed El Hassouni. Sonar image segmentation based on statistical modeling of wavelet subbands. ACS/IEEE International Conference on Computer Systems and Applications AICCSA 2015, Nov 2015, Marrakech, Morocco. hal-01266488

\section{HAL Id: hal-01266488 \\ https://hal.science/hal-01266488}

Submitted on 2 Feb 2016

HAL is a multi-disciplinary open access archive for the deposit and dissemination of scientific research documents, whether they are published or not. The documents may come from teaching and research institutions in France or abroad, or from public or private research centers.
L'archive ouverte pluridisciplinaire HAL, est destinée au dépôt et à la diffusion de documents scientifiques de niveau recherche, publiés ou non, émanant des établissements d'enseignement et de recherche français ou étrangers, des laboratoires publics ou privés. 


\title{
Sonar image segmentation based on statistical modeling of wavelet subbands
}

\author{
Ayoub KARINE ${ }^{1,3}$, Noureddine LASMAR ${ }^{3}$, Alexandre BAUSSARD ${ }^{3}$, Mohammed EL HASSOUNI ${ }^{1,2}$ \\ ${ }^{1}$ LRIT URAC 29, University of Mohammed V, Rabat, Morocco \\ 2 DESTEC, FLSHR, University of Mohammed V, Rabat, Morocco \\ \{ayoub.karine, mohamed.elhassouni\}@gmail.com \\ 3 ENSTA Bretagne / Lab-STICC (UMR CNRS 6285), \\ 2 rue François Verny, 29806 Brest Cedex 9 - France \\ \{noureddine.lasmar, alexandre.baussard\}@ensta-bretagne.fr
}

\begin{abstract}
This paper deals with the classification and segmentation of seafloor images recorded by sidescan sonar. To address this problem, related to texture analysis, a supervised approach is considered. The features of the textured images are extract by characterizing the wavelet coefficients through parametric probabilistic models. In this contribution, the generalized Gaussian distribution and the $\alpha$-stable distribution are used. For the classification step, two classifiers are considered: the k-nearest neighbor algorithm, that exploit the Kullback-Leibler divergence as similarity measurement, and the support vector machines. Experimental results on sonar images demonstrate the effectiveness of the proposed approach for sonar image classification and segmentation.
\end{abstract}

Keywords-Texture analysis, wavelet transform, statistical model, feature extraction, classification, segmentation, sonar images.

\section{INTRODUCTION}

This paper deals with the classification and segmentation of textured seafloor images recorded by sidescan sonar. Various methods have been already proposed in the literature to extract features from the textured sonar images for supervised or unsupervised classification purposes. For instance, they can be based on first order statistics [1], on second order statistics like Grey Level co-occurrence matrix [2,3] and methods based on spectral analysis via filter banks or transforms (Gabor filters, wavelet transforms, Fourier transform...) [4].

In this contribution, a conventional scheme of texture analysis, which consists in representing each wavelet subband by a parametric probabilistic model that represents the marginal statistics of subbands, is considered [5, 6]. In [7], it is suggested that the wavelet coefficients of sonar images can be best described by families of heavy-tailed distribution and considering the statistical model parameters in the wavelet domain as a salient feature of sonar images. Thus, in this contribution, we consider that our feature vector is composed by the parameters of the generalized Gaussian distribution (GGD) or by the parameters of the $\alpha$-stable distribution. Based on these feature vectors, two classifiers are studied: the knearest neighbor algorithm (KNN), that exploit the KullbackLeibler divergence (KLD) as similarity measurement, and the support vector machines (SVM).

This classification scheme is then exploited to segment sonar images. The proposed process is described in Figure1.
The two main steps are:

- The off-line phase: each image of a classified database is decomposed via a discrete wavelet transform (DWT). Using a prior model (GGD or $\alpha$-stable), the parameters of each wavelet subband are estimated and saved in a feature vector. These feature vectors are then used in the learning step.

- The on-line phase: classification/segmentation using a sliding window. The sonar image is divided into tiles of $L_{i m} \times L_{i m}$ pixels with a sliding step $p_{g}\left(p_{g}<<\right.$ $L_{i m}$ ) specified by the user (see Figure 2). The texture feature vector is calculated (in the wavelet domain) on each tile and used by the classifier. The classification of theses tiles leads to the segmented image.

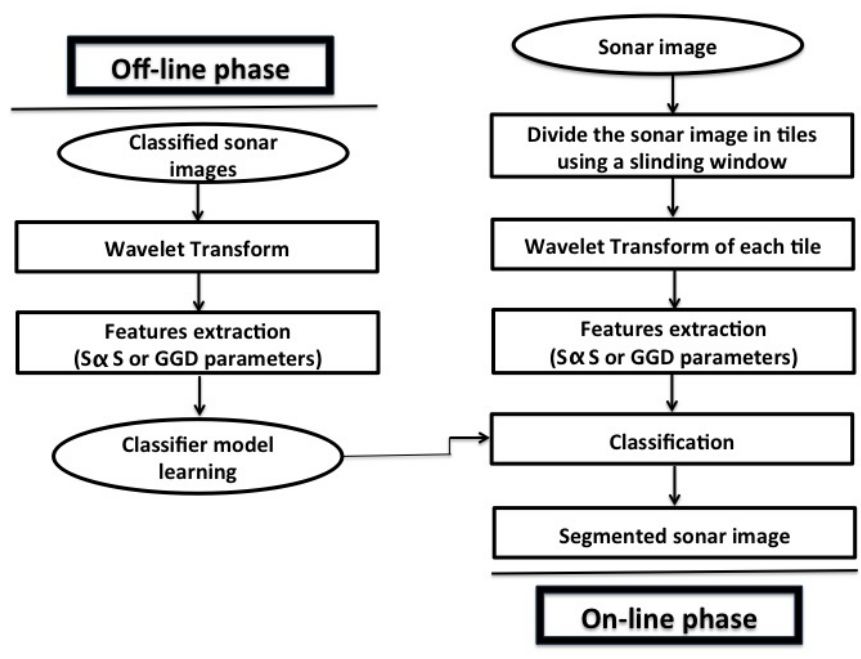

Fig. 1. Proposed segmentation method made of two steps: off-line phase and on-line phase.

The remainder of this paper is organized as follows. In section II the probabilistic models of the wavelet coefficients are introduced. Section III presents the two classifier considered in this contribution. In section IV, the experimental results are provided. Finally, section V gives some concluding remarks. 


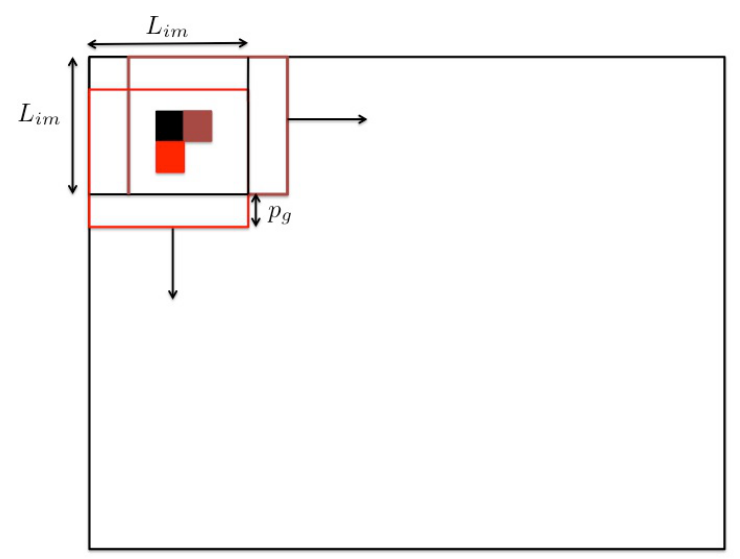

Fig. 2. Sliding window scheme of the image segmentation process.

\section{MOdELING THE WAVELET SUBBANDS}

As mentioned in the introduction, the GGD or the $\alpha$-stable parameters are used in this contribution to construct the feature vectors that describe the textures of the sonar images. In what follows, these models are briefly introduced.

\section{A. Alpha-stable distribution}

Due to lack of closed formulas for probability density function of the $\alpha$-stable, this distribution is best defined by its characteristic function as follows:

$\phi(\omega)=\left\{\begin{array}{l}\exp \left(j \mu \omega-|\gamma \omega|^{\alpha}\left[1-j \operatorname{sign}(\omega) \beta \tan \left(\frac{\pi \alpha}{2}\right)\right]\right), \alpha \neq 1 \\ \exp \left(j \mu \omega-|\gamma \omega|\left[1+j \operatorname{sign}(\omega) \beta \frac{2}{\pi} \ln (|\omega|)\right]\right), \alpha=1\end{array}\right.$

where $\alpha \in[0,2]$ is the characteristic exponent, $\beta \in[-1,1]$ is the skewness parameter, $\gamma>0$ is the dispersion parameter and $\mu \in \mathbb{R}$ is the location parameter. The latter four parameters can be estimated by using the McCulloch method [8].

In our study, it was found that the distribution of wavelet coefficients of our data is symmetric $(\beta=0)$ and centered at 0 $(\mu=0)$, then we use a symmetric and centered version of the $\alpha$-stable distribution ( $\mathrm{S} \alpha \mathrm{S})$. Consequently, the feature vector is composed by the estimated parameters as

$$
\text { Features }(\alpha-\text { stable })=\left(\alpha^{(j)}, \gamma^{(j)}\right)_{1 \leq j \leq 3 J},
$$

where $J$ is the number of scales.

\section{B. Generalized Gaussian distribution}

A random variable $X$ is distributed as generalized Gaussian if its probability density function (pdf) is given by

$$
f_{X}(x)=\frac{\beta}{2 \alpha \Gamma\left(\frac{1}{\beta}\right)} \exp \left[-\left(\frac{|x|}{\alpha}\right)^{\beta}\right] \quad x \in \mathbb{R}
$$

where $\alpha>0$ is the scale parameter, $\beta>0$ is the shape parameter and $\Gamma(z)=\int_{0}^{\infty} t^{z-1} e^{-t} d t$ is the Gamma function.
In this work, we use the Maximum-Likelihood method [6] in order to estimate these paramaters. The feature vector in this case is

$$
\text { Features }(G G D)=\left(\alpha^{(j)}, \beta^{(j)}\right)_{1 \leq j \leq 3 J},
$$

where $J$ is the number of scales.

\section{Similarity measurement}

In what follows, the classification problem is solved thanks to two classifiers. For the k-nearest neighbor method, a similarity measurement is needed. Thus, we propose to use the Kullback-Leibler divergence (KLD).

The KLD between two PDFs $\mathrm{P}$ and $\mathrm{Q}$ is given by

$$
K L D(P \| Q)=\int P(x) \ln \frac{P(x)}{Q(x)} d x .
$$

With the assumption of independence of wavelet coefficients in different subbands, the KLD between two images is the sum of KLDs between corresponding pairs of subbands.

$$
K L D\left(I_{1}, I_{2}\right)=\sum_{j=1}^{3 J} K L D(P \| Q),
$$

where $J$ is the number of scales.

1) KLD for the $S \alpha S$ : The KLD between two $\mathrm{S} \alpha \mathrm{S}$ distributions is not defined, but the KLD between two characteristic functions is defined in [9] as follows:

$$
\begin{array}{r}
K L D\left(\hat{\phi}_{1}\left(\alpha_{1}, \gamma_{1}\right) \| \hat{\phi}_{2}\left(\alpha_{2}, \gamma_{2}\right)\right)=\ln \left(\frac{c 2}{c 1}\right)-\frac{1}{\alpha_{1}}+ \\
\left(\frac{\gamma_{2}}{\gamma_{1}}\right)^{\alpha_{2}} \frac{\Gamma\left(\frac{\alpha_{2}+1}{\alpha_{1}}\right)}{\Gamma\left(\frac{1}{\alpha_{1}}\right)},
\end{array}
$$

where $c_{i}=\frac{2 \Gamma\left(\frac{1}{\alpha_{i}}\right)}{\alpha_{i} \gamma_{i}}, i=1,2$ and $\left(\alpha_{1}, \gamma_{1}\right),\left(\alpha_{2}, \gamma_{2}\right)$ are the $\mathrm{S} \alpha \mathrm{S}$ parameters of two subbands. Then the KLD between two images $I_{1}$ and $I_{2}$ is

$$
\begin{array}{r}
K L D_{S \alpha S}\left(I_{1}, I_{2}\right)=\sum_{j=1}^{3 J} K L D\left(\phi\left(\alpha_{1}^{(j)}, \gamma_{1}^{(j)}\right) \|\right. \\
\left.\phi\left(\alpha_{2}^{(j)}, \gamma_{2}^{(j)}\right)\right) .
\end{array}
$$

2) KLD for the GGD: Do and Vetterli [6] have defined a KLD between two GGD as follows:

$$
\begin{array}{r}
K L D\left(p\left(. ; \alpha_{1}, \beta_{1}\right) \| p\left(. ; \alpha_{2}, \beta_{2}\right)\right)=\ln \left(\frac{\beta_{1} \alpha_{2} \Gamma\left(\frac{1}{\beta_{2}}\right)}{\beta_{2} \alpha_{1} \Gamma\left(\frac{1}{\beta_{1}}\right)}\right) \\
+\left(\frac{\alpha_{1}}{\alpha_{2}}\right)^{\beta_{2}} \frac{\Gamma\left(\frac{\beta_{2}+1}{\beta 1}\right)}{\Gamma\left(\frac{1}{\beta_{1}}\right)}-\frac{1}{\beta_{1}},
\end{array}
$$

where $\left(\alpha_{1}, \beta_{1}\right)$ et $\left(\alpha_{2}, \beta_{2}\right)$ are the GGD parameters of two subbands. Consequently, the KLD between two images $I_{1}$ and $I_{2}$ is

$$
\begin{array}{r}
K L D_{G G D}\left(I_{1}, I_{2}\right)=\sum_{j=1}^{3 J} K L D\left(p\left(. ; \alpha_{1}^{(j)}, \beta_{1}^{(j)}\right) \|\right. \\
\left.p\left(. ; \alpha_{2}^{(j)}, \beta_{2}^{(j)}\right)\right),
\end{array}
$$


where $J$ is the number of scales.

\section{Classification Methods}

To classify the textured images two classifiers are considered and compared in this contribution: the SVM and the KNN.

\section{A. K-nearest neighbor method}

The KNN method [10] is based on the computation of the distance between the new observation and the observations of the learning database. It is laid out as described in Algorithm 1. In this contribution, a variation of the KNN classifier which uses the KLD as a similarity measure is considered $[9,11]$. It is also known as the probabilistic K-Nearest Neighbors (pKNN) classifier.

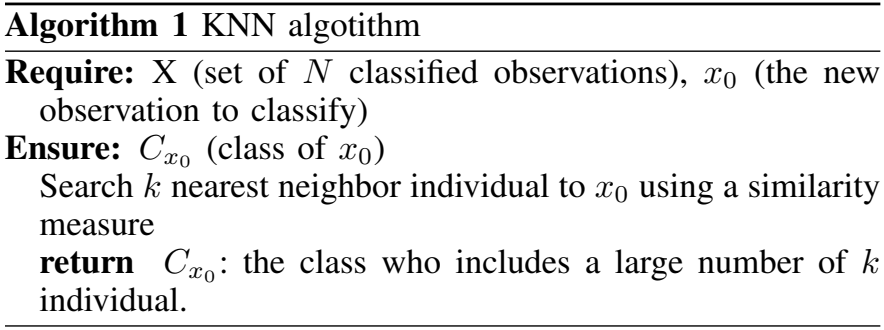

\section{B. Support vector machine}

Support vector machines are a binary classification method introduced by Vapnik [12]. They have strong theoretical foundations and excellent empirical successes. They have been applied to different applications such as SAR classifications [13], Face Recognition [14]...

The principle is to separate a given training set data with a hyperplane that is the maximally distant from them. The training instances that are closest to this hyperplane are called support vector. More generally, SVMs allow to project the original training data space to a higher dimensional feature space. The classification function is given as follow:

$$
f_{S V M}(x)=\sum_{x_{i} \in V S} \alpha_{i} y_{i} K\left(x, x_{i}\right)+b,
$$

where $V S$ are the vector supports, $\left(x_{i}, y_{i}\right)$ is the training set, $\alpha$ is the Lagrange multipliers obtained in the minimization process, $K$ is the kernel and $x$ is the observation to classify.

\section{EXPERIMENTAL RESULTS}

\section{A. Experiment data}

The database is composed of 3240 small images $(64 \times 64$ pixels). They have been extracted from sonar images recorded by a Klein 5500 sonar. These data have been provided by DGA/TN Brest. Each small image is labeled by its sea bottom type (Posidonia, ripples, rock, sand\&silt), the different classes are shown in Figure 3. Each class is composed of 810 small images.
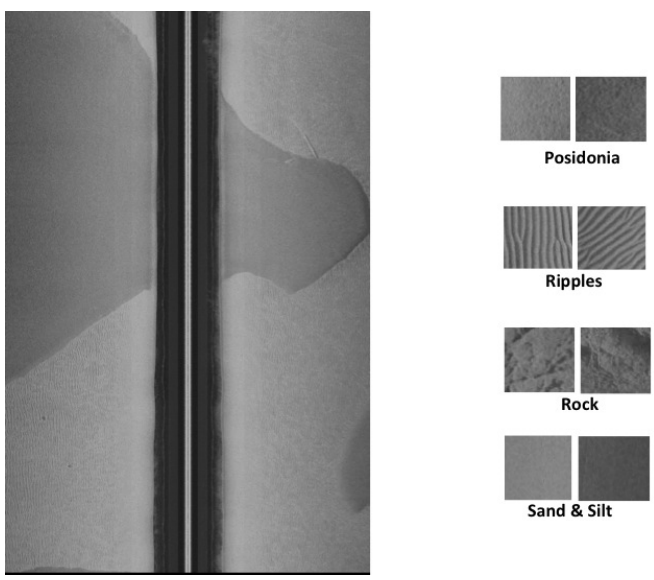

Fig. 3. Example of a sonar image and labeled small-images.

\section{B. Classification results}

In the on-line and the off-line processes two scales of the DWT (Daubechies 'db2' wavelet) are used for each small image. Then the $\alpha$-stable or the GGD distribution parameters are estimated to construct the feature vectors. Thus, these feature vectors are made of twelve parameters.

The leave-one-out cross validation is used for dividing the database into the training dataset and the validation dataset. For the KNN, an unknown observation is classified by assigning the label of the class to which the majority of its $k$ nearest neighbor belongs. The results in Figure 4 show that, for the GGD model, $k=3$ gives the highest classification accuracy. Note that similar results have been obtained using the $\mathrm{S} \alpha \mathrm{S}$ model.

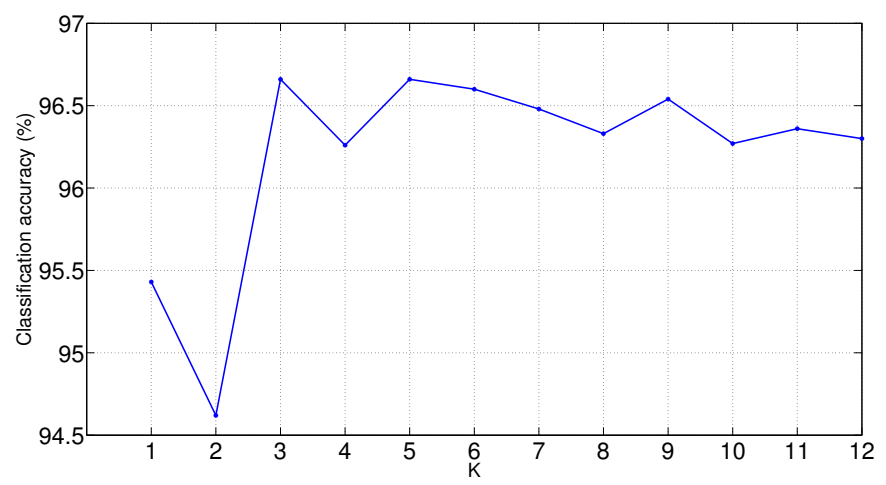

Fig. 4. Classification results, according to the values of $\mathrm{k}$, using the pknn approach and the GGD model.

For the SVM method, the well known libsvm [15] library is used. Table I gives some classification results according to the default value of each kernel.

\begin{tabular}{|l|l|}
\hline SVM Kernel & TC (\%) \\
\hline Linear & 89.88 \\
\hline Polynomial & 90.43 \\
\hline Gaussian & $\mathbf{9 0 . 5 6}$ \\
\hline Sigmoid & 42.01 \\
\hline
\end{tabular}

TABLE I. CLASSIFICATION RATE ACCORDING TO THE SVM KERNEL AND GGD. 
The radial basis function (RBF) is chosen, this kernel required two parameters $(c, \gamma)$ which can be selected with the cross-validation and grid-search as the following process:

- $\quad$ A grid space of $(c, \gamma)$ are considered with $c=2^{(-5: 15)}$ and $\gamma=2^{(-5: 15)}$,

- For each pair $(c, \gamma)$, conduct 2-fold cross-validation in the training set,

- Choose the pair $(c, \gamma)$ that has the best cross-validation accuracy,

- Create a classifier model using the best parameter.

Figure 5 shows classification results for each class according to the combination of the KNN and SVM classifiers, and the $\alpha$-stable and GGD features. The SVM combined with GGD leads to the highest classification rates for the different classes (92.59\% for posidonia, $96.20 \%$ for ripples, $100 \%$ for rock and $99.44 \%$ for sand\&silt) and for the average classification rate (97.31\%). Consequently, we select the SVM with RBF kernel $\left(C=2^{6}\right.$ and $\left.\gamma=2^{-4}\right)$ as classifier and the GGD model.

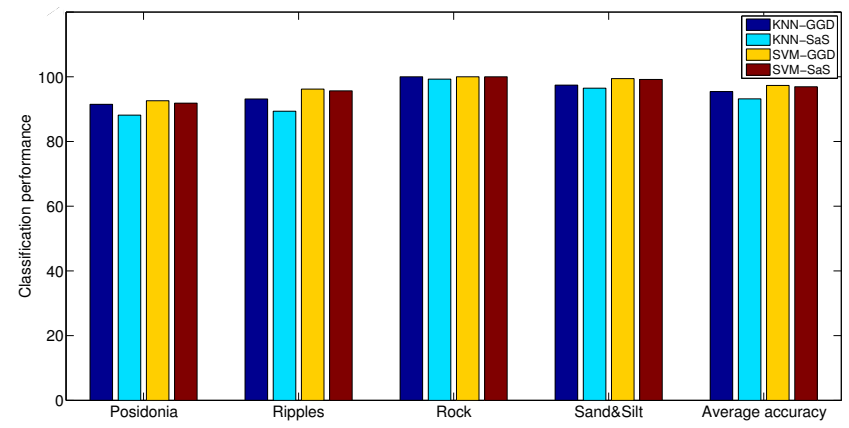

Fig. 5. Classification performances with different statistical models and different classifiers. II.

The corresponding confusion matrix is displayed in Table

\begin{tabular}{|l|l|l|l|l|}
\hline & Posidonia & Ripples & Rock & sand\&silt \\
\hline Posidonia & 92.59 & 2.22 & 0 & 5.18 \\
\hline Ripples & 1.94 & 96.20 & 0 & 1.85 \\
\hline Rock & 0 & 0 & 100 & 0 \\
\hline sand\&silt & 30.37 & 0.18 & 0 & 99.44 \\
\hline
\end{tabular}

TABLE II. CONFUSION MATRIX BY USING SVM AND GGD.

\section{Segmentation results}

The selected classification method and statistical model are now used in the on-line part of our process to segment sonar images. We note that the step of the sliding window used in our work is 8 . The results of the segmentation for different sonar images are shown in Figure 6. Despite the mislabelled of some pixels (essentially near the borders). It can be observed that different regions are perfectly distinguished. Note that, the errors of label at the border of two areas are probably due to a blocking effect. Indeed, the distribution parameters at a given pixel are estimated with wavelet coefficients from surrounding pixels, but data in this block do not always belong to a single class.
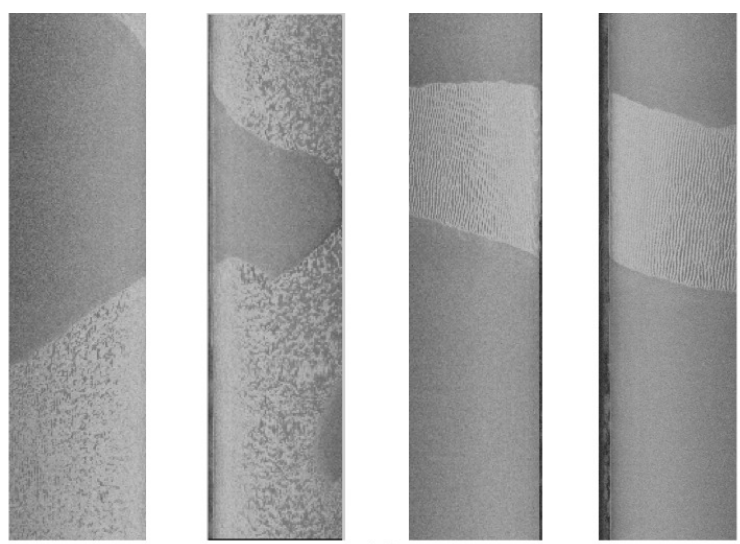

(a)
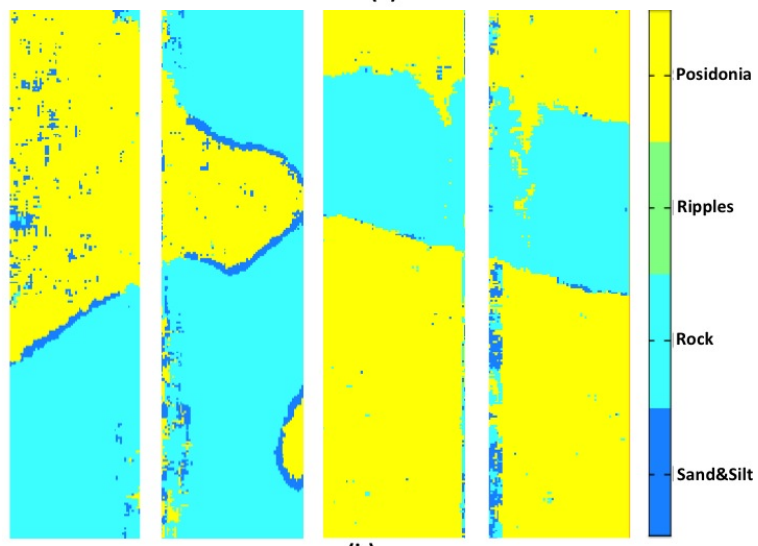

(b)

Fig. 6. Segmentation results using a proposed method. (a) The sonar image, (b) Segmentation result using SVM and GGD.

\section{Conclusion}

In this paper, a sonar image segmentation algorithm that take advantage of the statistics of the wavelet coefficients has been proposed. The $\alpha$-stable and the GGD parameters have been used to construct feature vectors used in the classification process based on KNN or SVM methods. The two models and the two classification approaches have been compared in order to select the best combination for the segmentation task. The first results proposed in this paper show the interest of the method. Several other numerical tests remain to be done using other databases in order to validate the interest and the robustness of the proposed method.

\section{REFERENCES}

[1] G. Le Chenadec and J.-M. Boucher, "Sonar image segmentation using the angular dependence of backscattering distributions," in Proceedings of IEEE/MTS OCEANS, Brest, 2005.

[2] I. Karoui, R. Fablet, J.-M. Boucher, and J.-M. Augustin, "Seabed segmentation using optimized statistics of sonar textures," IEEE Journal of Oceanic Engineering, vol. 47, no. 6, pp. 1621-1631, 2009.

[3] M. Lianantonakis and Y. Petillot, "Sidescan sonar segmentation using texture descriptors and active contours," IEEE Journal of Oceanic Engineering, vol. 32, no. 3, pp. 744-752, 2007. 
[4] A. Nait-Chabane, B. Zerr, and G. Le Chenadec, "Spectral directional filter bank for sidescan sonar segmentation with unsupervised neural network approach," in International Conference and Exhibition on Underwater Acoustics, Corfu, 2013.

[5] S. Mallat, "A theory for multiresolution signal decomposition: the wavelet representation," IEEE Transactions on Pattern Analysis and Machine Intelligence, vol. 11, no. 7, pp. 674-693, Jul. 1989.

[6] M. Do and M. Vetterli, "Wavelet-based texture retrieval using generalized gaussian density and KullbackLeibler distance," IEEE Transactions on Image Processing, vol. 11, no. 2, pp. 146-158, 2002.

[7] N.-E. Lasmar, A. Baussard, and G. Le Chenadec, "Seafloor classification using statistical modeling of wavelet subbands," in 2nd International Conference and Exhibition on Underwater Acoustics (UAC 2014), Rhodes, Greece, Jun. 2014.

[8] J. H. McCulloch, "Simple consistent estimators of stable distribution parameters," Communications in Statistics Simulation and Computation, vol. 15, no. 4, pp. 11091136, 1986.

[9] E. Kornaropoulos and P. Tsakalides, "A novel knn classifier for acoustic vehicle classification based on alphastable statistical modeling," in Statistical Signal Processing, 2009. SSP '09. IEEE/SP 15th Workshop on, Aug 2009, pp. 1-4.

[10] T. Cover and P. Hart, "Nearest neighbor pattern classification," IEEE Transactions on Information Theory, vol. 13, no. 1, pp. 21-27, January 1967.

[11] N.-E. Lasmar, A. Baussard, and G. L. Chenadec, "Asymmetric power distribution model of wavelet subbands for texture classification," Pattern Recognition Letters, vol. 52, pp. $1-8,2015$.

[12] V. N. Vapnik, The Nature of Statistical Learning Theory. Springer-Verlag New York, USA, 1995.

[13] Y. Peng, X. Xu, W. Zhou, and Y. Zhao, "SAR image classification based on alpha-stable distribution," Remote Sensing Letters, vol. 2, no. 1, pp. 51-59, 2011.

[14] E. Osuna, R. Freund, and F. Girosi, "Training support vector machines: an application to face detection," in IEEE Computer Vision and Pattern Recognition. IEEE, 1997, pp. 130-136.

[15] C.-C. Chang and C.-J. Lin, "LIBSVM: A library for support vector machines," ACM Transactions on Intelligent Systems and Technology, vol. 2, pp. 27:1-27:27, 2011, software available at http://www.csie.ntu.edu.tw/ cjlin/ libsvm. 